\title{
Toxic Trauma: A Basic Clinical Guide, Second Edition
}

\section{INTRODUCTION}

This leading comprehensive book is considered as one of the newest books among distinguished scholars in medicine. The book is aimed to ease off the problems related to toxic attacks and toxic chemicals. Moreover, the book is a powerful means of disseminating information among practitioners and paramedicines in medical services. This is the second edition of the book, first published in 2014. Toxic Trauma is thought of as an uncommon cause of Emergency Department (ED), Because of that, medicine physicians and emergency medical technicians encounter difficulties in dealing with this trauma on their optimal management. This book offers experimental guidance to emergency personnel and emergency medicines and paramedical personnel in the event of having to manage separable or mass chemical casualties as part of their work in a neutral ambulance service or hospital. The text sheds some light on the nature and basic science of the hazards faced as well as the practical management of people exposed to chemicals and toxins. All chapters are devoted to cover the development and classification of chemical toxic agents, how exposure can happen and how medical staff should be involved in its management.

There is also the meticulous consideration of the mother and source of toxic trauma and the pathophysiological procedures involved, together with an organised method of early and ongoing management reinforced by detailed examples of incidents. This guide is a beneficial applied resource for emergency medical and paramedical personnel involved in the management of mass chemical victims and with warranting safe operating procedures within possibly contaminated areas to prevent secondary victims from a chemical release. Dr. Baker has dealt with these problems in this new edition of Toxic Trauma. He has drawn out and sophisticated some concepts of supportive and specific therapy by describing a sequences of toxidromes (clinical syndromes in relation to exposure to toxic materials) that portray the effects of a number of groups of toxic materials.

A key strength of this book is that it extensively elucidates the major concerns in toxic trauma and its impacts on victims in a more detailed description. Toxic Trauma: A Basic Clinical Guide has valuable information for students wanting to examine the subject areas covered in more depth and keep the information up-to-date. Overall, this book is eminently appropriate for academics and students in medical studies. Moreover, the book does add new concepts and offers a deeper understanding of toxic trauma. This textbook undoubtedly has virtue in the medical literature and will be of value to academics and researchers alike.

As the current book is thought of a comprehensive useful book about Toxic Trauma, it is suggested that the author can shed more light on the staff behaviour during treatment, including training staff in order to behave effectively with Toxic Trauma patients and managing their treatment particularly in the hospital and prehospital level.

\section{PARTICULARS OF CONTRIBUTORS:}

1. Department of Critical Care Nursing, School of Nursing (Aligoodarz) Lorestan University of Medical Science, Lorestan, Iran.

NAME, ADDRESS, E-MAIL ID OF THE CORRESPONDING AUTHOR:

Dr. Amin Talebi,

Department of Critical Care Nursing, School of Nursing (Aligoodarz) Lorestan University of Medical Science, Lorestan, Iran.

E-mail: talebiamin5@gmail.com

Date of Submission: Jun 13, 2018 Date of Peer Review: Jul 24, 2018 Date of Acceptance: Aug 24, 2018 Date of Publishing: Nov 01, 2018 